\title{
Femoral nerve decompression and sartorius-to-quadriceps nerve transfers for partial femoral nerve injury: a cadaveric study and early case series
}

\author{
Colin W. McInnes, MD, ${ }^{1}$ Austin Y. Ha, MD, ${ }^{2}$ Hollie A. Power, MD, ${ }^{3}$ Thomas H. Tung, MD, ${ }^{2}$ and \\ Amy M. Moore, MD4
}

\begin{abstract}
'Department of Surgery, Division of Plastic Surgery, Fraser Health Authority/University of British Columbia, New Westminster, British Columbia, Canada; '2Department of Surgery, Division of Plastic and Reconstructive Surgery, Washington University School of Medicine in St. Louis, Missouri; ${ }^{3}$ Department of Surgery, Division of Plastic Surgery, University of Alberta, Edmonton, Alberta, Canada; and ${ }^{4}$ Department of Plastic and Reconstructive Surgery, The Ohio State University Wexner Medical Center, Columbus, Ohio
\end{abstract}

\begin{abstract}
OBJECTIVE Partial femoral nerve injuries cause significant disability with ambulation. Due to their more proximal and superficial location, sartorius branches are often spared in femoral nerve injuries. In this article, the authors report the benefits of femoral nerve decompression, demonstrate the feasibility of sartorius-to-quadriceps nerve transfers in a cadaveric study, describe the surgical technique, and report clinical results.
\end{abstract}

METHODS Four fresh-frozen cadaveric lower limbs were dissected for anatomical analysis of the sartorius nerve. In addition, a retrospective review of patients with partial femoral nerve injuries treated with femoral nerve decompression and sartorius-to-quadriceps nerve transfers was conducted. Pre- and postoperative knee extension Medical Research Council (MRC) grades and pain scores (visual analog scale) were collected.

RESULTS Up to 6 superficial femoral branches innervate the sartorius muscle just distal to the inguinal ligament. Each branch yielded an average of 672 nerve fibers (range 99-1850). Six patients underwent femoral nerve decompression and sartorius-to-quadriceps nerve transfers. Four patients also had concomitant obturator-to-quadriceps nerve transfers. At final follow-up (average 13.4 months), all patients achieved MRC grade 4-/5 or greater knee extension. The average preoperative pain score was 5.2 , which decreased to 2.2 postoperatively $(p=0.03)$.

CONCLUSIONS Femoral nerve decompression and nerve transfer using sartorius branches are a viable tool for restoring function in partial femoral nerve injuries. Sartorius branches serve as ideal donors in quadriceps nerve transfers because they are expendable, are close to their recipients, and have an adequate supply of nerve fibers.

https://thejns.org/doi/abs/10.3171/2020.6.JNS20251

KEYWORDS femoral nerve injury; nerve to sartorius; nerve decompression; nerve transfer; cadaveric study; peripheral nerve

$\mathrm{Q}$ UADRICEPS muscle function is critical for normal, unassisted ambulation without the use of braces or splints. Patients with femoral nerve injury have an impeded gait and are often unable to run or climb stairs. Possible etiologies include trauma, malignancy, virus-mediated phenomena, or, most commonly, iatrogenic injury. ${ }^{1}$

Methods to restore quadriceps muscle function include direct nerve repair, nerve grafting, tendon transfers, functional muscle transfers, and prosthetics. Choices for intervention and outcomes are dependent on the time from injury, etiology, and prognosis for recovery. ${ }^{1-7}$ Nerve transfers are a viable option when the injury is proximal and access to the proximal stump is difficult. The benefits of nerve transfers are well established: they provide a source of viable axons closer to the target muscle than nerve grafts, and, compared with tendon transfers, they reinnervate the native muscles, leading to an optimized length/tension ratio, more natural function, and potentially greater strength. Nerve transfers also avoid the need for extended postoperative immobilization. Obturator branches have been previously described as suitable nerve transfer donors for reconstruction of complete femoral nerve palsy. ${ }^{8}{ }^{8}$ From a technical standpoint, there are two main types of nerve transfers utilized to restore

ABBREVIATIONS EMG = electromyography; ETE = end-to-end; MRC = Medical Research Council; MUAP = motor unit action potential; ROM = range of motion; SETS = supercharged end-to-side.

SUBMITTED January 24, 2020. ACCEPTED June 30, 2020.

INCLUDE WHEN CITING Published online November 6, 2020; DOI: 10.3171/2020.6.JNS20251. 
motor function. An end-to-end (ETE) nerve transfer is intuitive; the distal end of the nerve from the donor muscle is coapted to the proximal end of the recipient nerve. The downside to utilizing an ETE transfer for a partial nerve injury is that it requires the partially injured nerve to be transected, eliminating the possibility of any native recovery within that nerve.

The supercharged end-to-side (SETS) transfer, also referred to as a reverse end-to-side transfer, is a surgical technique to augment function of a regenerating nerve while allowing native regeneration to occur. To date, the SETS transfer has not yet been described for femoral nerve injuries. ${ }^{10}$ SETS transfers involve creating an epineural window and sewing the transferred nerve to the side of an otherwise intact nerve. Studies have demonstrated that the transferred nerve will regenerate down the injured nerve to the new target muscle. ${ }^{11,12}$ SETS transfers have also been studied clinically for both acute traumatic nerve injury and compression neuropathy and are an increasingly utilized option. ${ }^{10-15}$

In previous femoral nerve decompression and nerve transfer procedures for femoral nerve injuries, we observed that the sartorius branches were often spared. We noted this pattern by stimulating branches of the femoral nerve with a handheld nerve stimulator. Given the relative expendability of the sartorius nerve branches and their proximity to other femoral nerve branches, we began exploring their use as donors for restoring quadriceps muscle function. The objective of this article was to demonstrate the importance of femoral nerve decompression and the feasibility of sartorius-to-quadriceps nerve transfers in a cadaveric study, describe the surgical technique in patients with partial femoral nerve injuries, and report early clinical results.

\section{Methods}

\section{Part A: Cadaveric Study}

Four fresh-frozen cadaveric lower limbs were dissected for anatomical analysis of the femoral nerve and its branches to the sartorius. Surface anatomy landmarks, branching patterns, and nerve fiber counts of terminal branches greater than $1 \mathrm{~mm}$ in diameter were recorded. The methodology of nerve sample processing and histological analysis used in this study has been previously reported ${ }^{16,17}$ Briefly, the nerves were fixed in 3\% glutaraldehyde solution in 0.1 $\mathrm{M}$ phosphate-buffered saline $(\mathrm{pH} 7.2)$ at $4^{\circ} \mathrm{C}$ prior to sectioning. The tissues were then postfixed with $1 \%$ osmium tetroxide and serially dehydrated in ethanol. Specimens were embedded in Araldite 502 (Polysciences Inc.), sectioned into $1-\mu \mathrm{m}$-thick cross-sections, and stained with $1 \%$ toluidine blue and evaluated via light microscopy. Microscopic images were analyzed with an automated digital image analysis system linked to histomorphometry software to quantify the total nerve fiber count.

\section{Part B: Retrospective Case Series}

After obtaining institutional review board approval, a retrospective review of all patients with quadriceps muscle weakness treated with femoral nerve decompression and sartorius-to-quadriceps nerve transfers by the

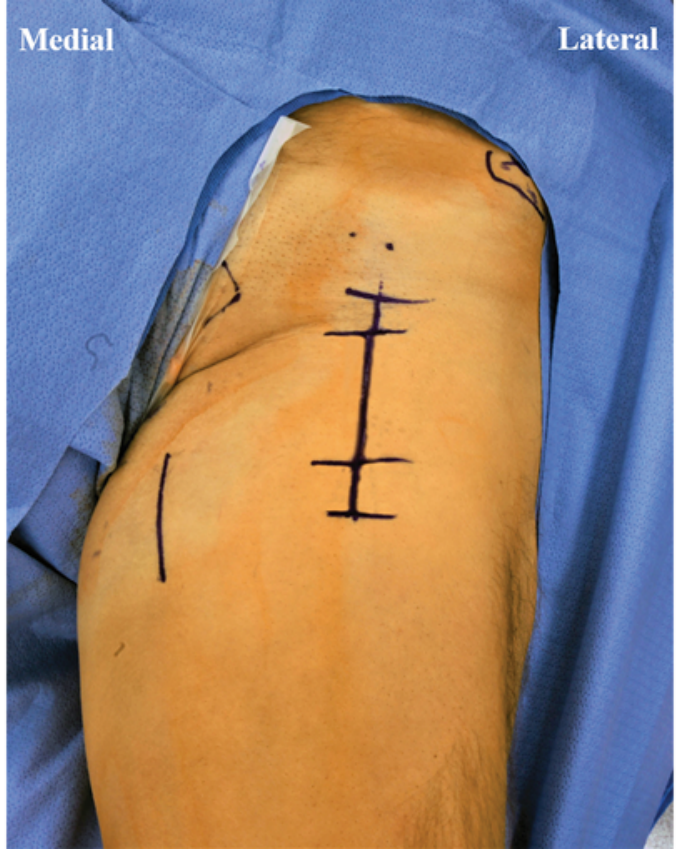

FIG. 1. Incision for femoral nerve decompression and sartorius-to-femoral nerve transfers. Figure is available in color online only.

senior authors (T.H.T. and A.M.M.) was conducted. Patients with virus/immune-mediated femoral nerve palsy (e.g., acute flaccid myelitis) and patients with less than 3 months of postoperative follow-up were excluded. Preoperative workup included electromyography (EMG) testing of the quadriceps muscles to determine the extent of native recovery, if any. This was completed by neurologists with fellowship training in electrodiagnostic testing. We do not perform EMG of the sartorius muscle and instead rely on intraoperative assessment with a nerve stimulator. Pre- and postoperative Medical Research Council (MRC) strength and pain scores were evaluated. Descriptive statistics were calculated. Normality of continuous variables was assessed using the Shapiro-Wilk test. A two-tailed, paired Student t-test was used to compare pre- and postoperative pain scores (version 24.0, IBM SPSS Statistics, IBM Corp.).

\section{Surgical Technique}

The patient is positioned supine on the operating table with the hip in partial external rotation. An 8-cm longitudinal incision is marked just below the inguinal crease and lateral to the palpable femoral artery (Fig. 1). This corresponds to a point about two-thirds of the distance from the pubic tubercle to the anterior superior iliac spine. Decompression of the femoral nerve requires release of 3 layers of investing fascia: the superficial fascia, the fascia of the sartorius muscle, and the femoral sheath. After incising through the superficial fascia within the subcutaneous fat, dissection continues to the muscular fascia of the sartorius muscle, which is incised over the medial border (Fig. 2 left). The sartorius muscle is then retracted laterally, and its motor nerves can be seen entering the muscle on the 


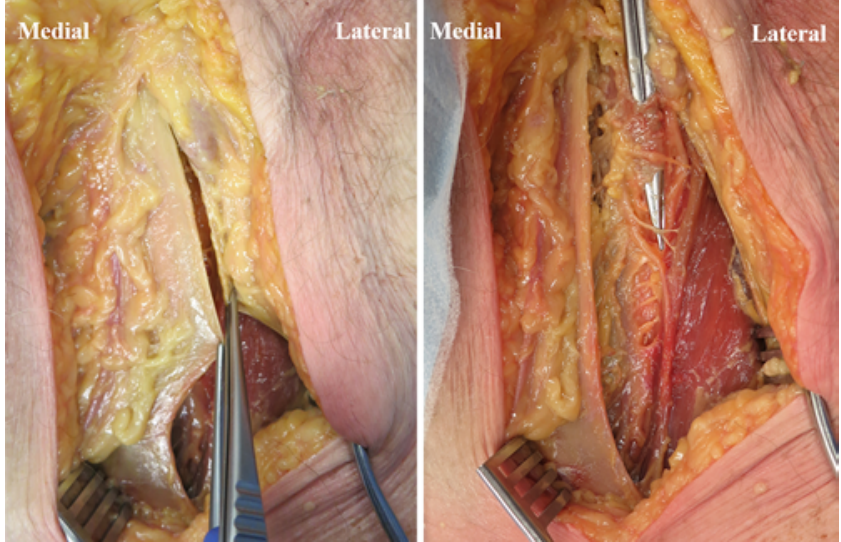

FIG. 2. Left: Fascia overlying the sartorius muscle. Right: Femoral sheath. Both are incised to expose the femoral neurovascular bundle and nerve branches to the sartorius muscle. Figure is available in color online only.

deep surface of its medial border. These nerve branches are superficial relative to the quadriceps nerve branches.

The femoral sheath is then incised overlying the femoral neurovascular bundle, distal to the inguinal ligament and lateral to the palpable vessels (Fig. 2 right). The sartorius branches are traced proximally to the femoral nerve trunk. Exposure and dissection of the femoral vessels should be avoided, as this may disrupt lymphatic drainage. Once the femoral nerve trunk is identified, the fascia overlying the nerve branches proximally and distally along the nerve, freeing it from any scar tissue. The inguinal ligament is identified and retracted. Complete decompression is achieved when it is possible to guide a finger over the femoral nerve into the retroperitoneal space (Fig. 3). Release of the inguinal ligament is not required for a complete decompression.

The femoral nerve branches are then neurolyzed distally to their insertions. They are individually stimulated

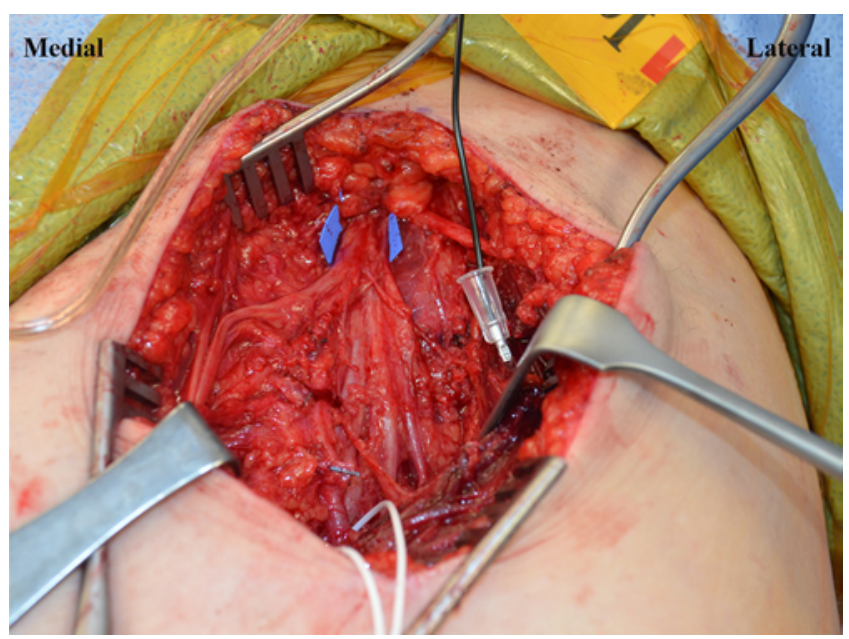

FIG. 3. The femoral nerve trunk after complete decompression. The sartorius muscle is being retracted laterally. Figure is available in color online only.

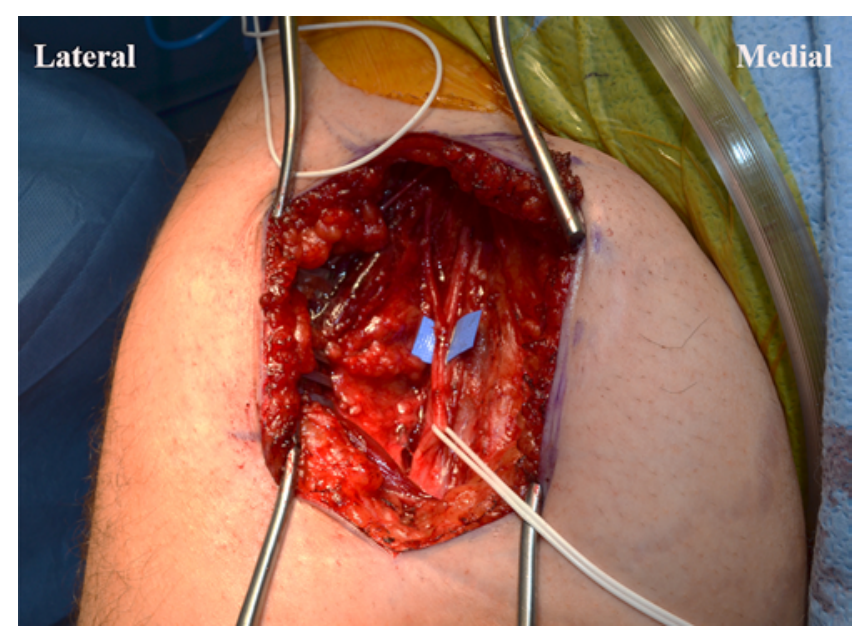

FIG. 4. Patient 4. Intraoperative photograph showing a right sartoriusto-vastus medialis SETS nerve transfer. Figure is available in color online only.

with a handheld nerve stimulator (Checkpoint Surgical, Inc.) to assess function. The sartorius branches must exhibit strong response to stimulation at $0.5 \mathrm{~mA}$ and $100 \mathrm{~Hz}$ to be deemed appropriate donors. An ETE nerve transfer is completed in cases in which the recipient nerve has no response (i.e., no visible contraction) and preoperative EMG shows no motor unit action potentials (MUAPs). An SETS nerve transfer is completed if there is at least partial preservation of the recipient function and if there is evidence of available motor endplates on preoperative EMG in the form of fibrillations and positive sharp waves (Fig. 4). When possible, we preserve 1-2 sartorius branches to maintain sartorius function.

To perform SETS transfers, the donor sartorius nerve branches are transected distally and brought to the recipient nerve branches. The lower extremity is then taken through a full range of motion (ROM) to ensure tensionfree repair. Epineurial and perineurial windows are then created in the recipient nerve at these locations. The window size should match the caliber of the donor nerve. Four simple interrupted sutures are placed under microscope magnification using 9-0 nylon suture, and fibrin glue is used to reinforce the coaptation. A closed-suction drain is placed and removed at 1 week postoperatively. A knee immobilizer is used for 3 weeks postoperatively. Weight bearing is allowed as tolerated. Physical therapy is initiated at the time of initial evaluation of patients with lowerextremity nerve injuries to ensure supple joints with maximum passive ROM and continued in the postoperative period for donor activation-focused rehabilitation. ${ }^{18}$

\section{Results}

\section{Part A: Cadaveric Study}

The femoral nerve was located 63\% (range 59\%-67\%) of the distance from the pubic tubercle to the anterior superior iliac spine. Five to six nerve branches greater than $1 \mathrm{~mm}$ in diameter innervated the sartorius muscle in its 


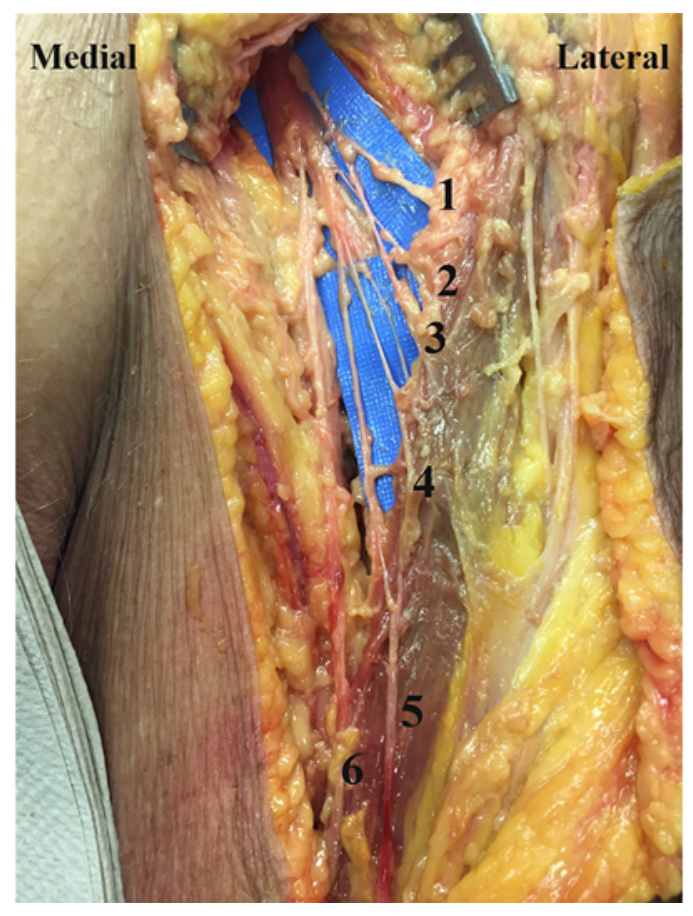

FIG. 5. Cadaveric dissection of the left thigh showing a typical branching pattern of the sartorius nerve branches. Figure is available in color online only.

proximal third, all branching from the femoral nerve at or just proximal to the inferior aspect of the inguinal ligament (Fig. 5). The first branch innervated the muscle 5 $\mathrm{cm}$ (range 4-7 cm) below the inguinal ligament and the most distal branch entered $12 \mathrm{~cm}$ (range 11-14 cm) below. Histological analysis of 25 sartorius nerve branches showed an average of 672 nerve fibers (range 99-1850) in each branch. The sartorius branches were always the most superficial of the femoral nerve branches. The most distal sartorius branch continues through the sartorius to the overlying skin as a sensory branch. All branches could be transposed onto the quadriceps femoral branches without tension.

\section{Part B: Retrospective Case Series}

Between August 2017 and February 2019, 6 patients with partial femoral nerve palsy underwent femoral nerve decompression and sartorius nerve transfers for knee extension. Patient 5 underwent an identical procedure in the bilateral lower extremities. The data are summarized in Table 1.

Three of the $6(50 \%)$ patients were male, and the average age at time of surgery was 46.8 years (range 14-69 years). The average interval from injury to surgery was 8.6 months (range $6-15$ months), and the average followup was 13.4 months (range 4-24 months). Etiology of the injury included gunshot wounds $(2 / 6,33.3 \%)$, iliopsoas hematoma and compartment syndrome $(2 / 6,33.3 \%)$, and iatrogenic $(2 / 6,33.3 \%)$.

The preoperative knee extension MRC grade was $2 / 5$ in 4 patients (66.7\%) and $3 / 5$ in 2 patients (33.3\%). All had full

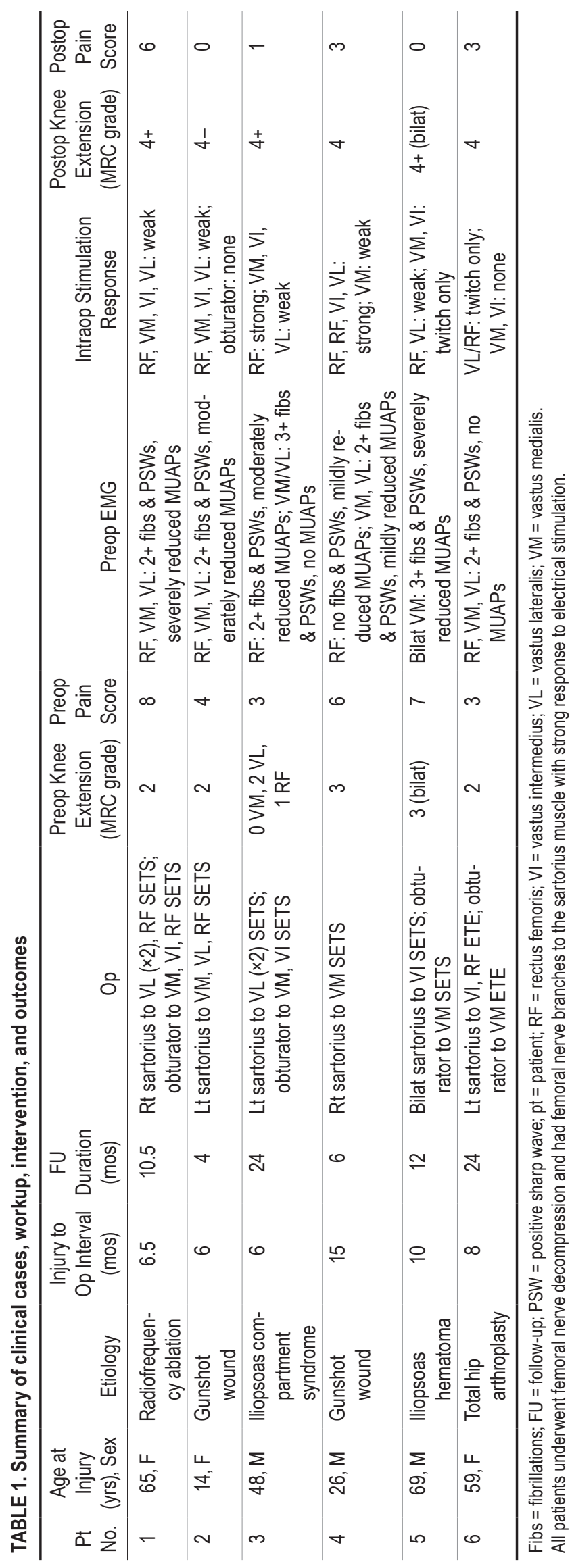



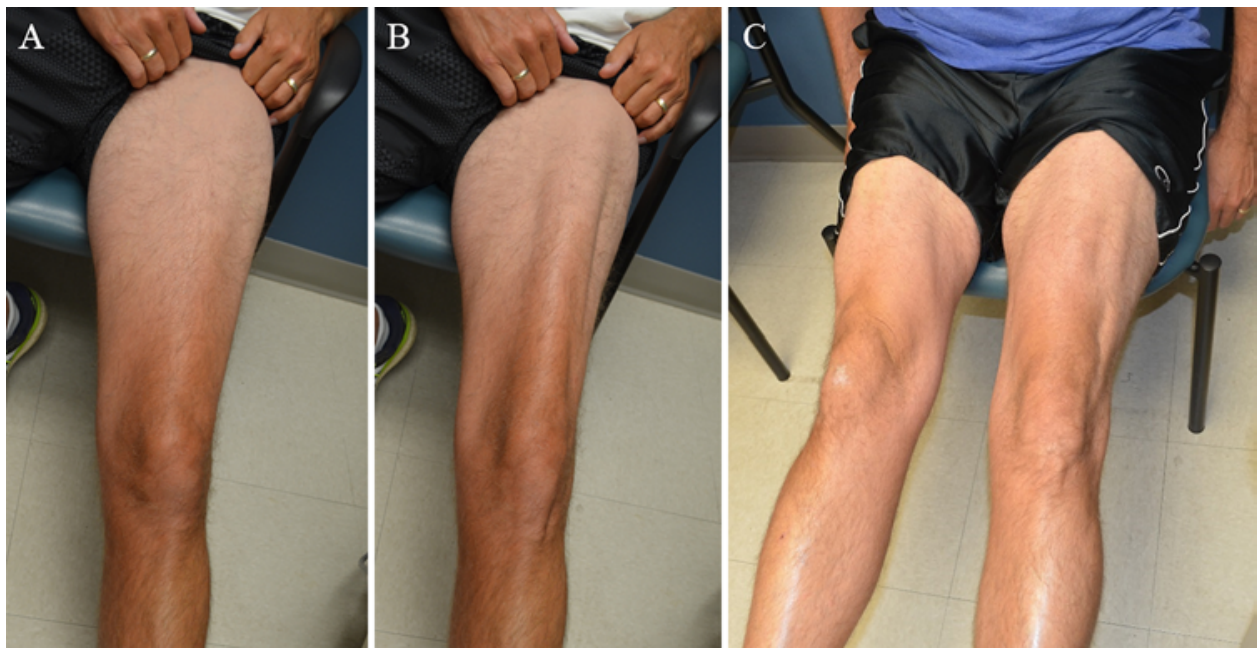

FIG. 6. Patient 3. A and B: Photographs obtained at 6 months after femoral nerve injury from iliopsoas compartment syndrome, showing atrophy and weak contraction of his rectus femoris and no contraction of the vastus medialis and lateralis. C: The same patient at 12 months postoperatively with full extension of the knee with firing of the rectus femoris, vastus medialis, and vastus lateralis muscles as well as improved muscle bulk in all quadriceps muscles. Figure is available in color online only.

passive ROM but required bracing, and 4 required assist devices (crutches and/or wheeled walker) for ambulation preoperatively. All patients achieved MRC grade 4-/5 or greater postoperatively and subjectively noted improved strength, increased muscle bulk, and more natural gait over time (Fig. 6). None were using braces or assist devices at the final follow-up. The average preoperative pain score was 5.2 (range 3-8), which decreased to 2.2 (range $0-6)$ postoperatively $(\mathrm{p}=0.03)$. Preoperative EMG was completed in all patients and demonstrated fibrillations and positive sharp waves in one or more of the quadriceps muscle groups. The presence of fibrillations and positive sharp waves indicates the availability of unoccupied motor endplates that can be reinnervated..$^{10-12,14}$ In all patients, EMG testing also showed reduced, but present, MUAPs in one or more quadriceps muscles, indicating a partial nerve injury.

The results of intraoperative stimulation can be found in Table 1. A strong response of the sartorius muscle is a requirement for its use as a donor, and this was present in all 6 patients. In cases in which there was at least a weak response to electrical stimulation, an SETS nerve transfer was completed to preserve and augment the native motor nerve function. In patient 6 , whose quadriceps muscle group had no response to electrical stimulation, an ETE nerve transfer was completed. In 4 patients, obturator-toquadriceps nerve transfers were also performed. Patient 2 was not a candidate for this transfer, as her obturator nerve was also injured and did not respond to electrical stimulation. Patient 4 had a strong response in all quadriceps muscles except for the vastus medialis, and the sartorius transfer alone was deemed adequate to augment its function.

\section{Discussion}

Intact quadriceps muscle function is essential for unassisted, independent ambulation without the use of splints, braces, or other devices. As such, it is critical to restore its function in the setting of a femoral nerve palsy. Nerve transfers offer several advantages over tendon transfers, including the potential to reinnervate the native quadriceps for ultimately stronger and more natural function, reduced donor site morbidity, and shorter immobilization postoperatively. ${ }^{6}$ Our group has previously reported early results of obturator-to-femoral nerve transfers. ${ }^{9}$ In this article, we demonstrate the feasibility of sartorius-toquadriceps nerve transfers in a cadaveric study, describe the surgical technique for femoral nerve decompression and nerve transfers in patients with partial femoral nerve injuries, and report clinical results.

\section{Anatomy of the Sartorius Nerve Branches}

Cadaver dissection revealed 5-6 branches of the femoral nerve to the sartorius muscle with a diameter of $1 \mathrm{~mm}$ or greater, which is consistent with the findings of Tanaka et al. ${ }^{19}$ Nerve branches innervated the muscle on the deep surface of its medial border and were accompanied by small vascular branches. A previous study demonstrated that the sartorius muscle was innervated by 1-2 main branches arising from the femoral nerve with an average length of $10 \mathrm{~cm}$ (range 5-14 cm) and an average diameter of $1.7 \mathrm{~mm}$ (range $0.7-2.5 \mathrm{~mm}$ ). ${ }^{20}$ More recently, Buckland et al. reported there were $1(40 \%)$ or $2(60 \%)$ main nerve branches that arborized closer to the muscle,${ }^{21}$ findings similar to our study and others..$^{19,22}$

\section{Suitability of the Sartorius as a Donor Nerve}

The sartorius muscle is a secondary hip flexor, thigh adductor, and knee flexor, and is widely considered expendable. ${ }^{21}$ It is commonly used in reconstructive surgery as a muscle flap for small groin wounds, often following vascular bypass surgery. Although the sartorius muscle contributes to knee flexion through its insertion at the pes anserinus, it has been used successfully for enhancing knee extension as a tendon transfer. ${ }^{6}$ It was found to 
adapt and function in phase with the remaining quadriceps muscles because of its common origin of innervation from the femoral nerve. ${ }^{23}$ This finding supports its use as a donor for quadriceps reinnervation and in many ways facilitates the donor activation-focused rehabilitation after the nerve transfer. ${ }^{18}$

Potentially due to its more proximal and superficial branching location, sartorius branches are often spared in partial femoral nerve injuries. They are also close to the quadriceps branches, which allows for a graft-free and tension-free coaptation through all ranges of motion of the lower extremity. Lastly, our histological analysis suggests that the sartorius branches supply enough nerve fibers to power the larger quadriceps muscles. In this sample of 25 sartorius nerve branches, an average of 672 nerve fibers (range 99-1850) were present in each branch. This is comparable to the 1019 fibers present in the branch to the vastus medialis muscle and 455 fibers present in the branch to the vastus lateralis muscle, as reported by Moore et al. ${ }^{16}$ This suggests that there are abundant axons available to restore partial femoral nerve injuries. If none of the quadriceps muscles stimulate after decompression and sartorius branches are preserved, they can be utilized in conjunction with obturator nerve branches to add additional axons to the muscles dedicated to knee extension. If there is a partial femoral nerve injury (e.g., clinical weakness, signs of limited recovery to specific branches), sartorius branches may be utilized alone in an end-to-side fashion with no morbidity.

\section{Clinical Results}

The patients in this series all had substantial quadriceps muscle weakness preoperatively that significantly improved following femoral nerve decompression and sartorius-to-quadriceps nerve transfers. Four patients also underwent concomitant obturator-to-quadriceps nerve transfers. The average interval from injury to surgery was 9 months, suggesting that these patients had meaningful knee extension that failed to recover spontaneously, and the improvement seen postoperatively is due to the surgical intervention. Postoperatively, all patients achieved a knee extension MRC grade of $4-/ 5$ or greater and noted improved strength, increased muscle bulk, and more natural gait over time (Video 1).

VIDEO 1. Patient 3. Part 1: The patient has an abnormal preoperative gait and weak contraction of the rectus femoris only. Part 2:

The same patient jogging at 12 months postoperatively with restoration of more normal gait and improved strength. Copyright Amy M.

Moore. Published with permission. Click here to view.

The decrease in postoperative pain scores was also statistically significant. These improvements were seen despite the variability in the etiology of femoral nerve injury and patient age and sex.

We attribute improvement of function in our patients to both the femoral nerve decompression and the nerve transfers; however, it is difficult to ascertain the exact contribution of each component. A subgroup of patients, more specifically, patient 4 in this series, experienced an immediate difference postoperatively. This is likely due to the resolution of ischemic conduction block within the femoral nerve. In contrast, sustained long-term improvement in function and especially muscle bulk are more likely a result of nerve transfers, as the regeneration of nerve fibers reinnervates previously unoccupied motor endplates, leading to muscle contraction and hypertrophy.

\section{Treatment Algorithm}

Our treatment algorithm for all nonsharp injuries to the femoral nerve, such as blunt trauma, thermal injuries, and gunshot wounds, typically involves a period of monitoring for spontaneous recovery with serial physical examination and electrodiagnostic testing starting at 3 months postinjury. If there is delayed return of function or no evidence of spontaneous recovery (e.g., no MUAPs) on electrodiagnostic testing, we plan for operative intervention by 6 months. At minimum, all patients undergo decompression of the femoral nerve. Whether they are candidates for nerve transfers, and if so, which type of transfer, depends on findings on their preoperative EMG study and intraoperative nerve stimulation, as stated above.

Based on our understanding of nerve transfers in the upper extremity, we interpret the presence of fibrillations and positive sharp waves on EMG as evidence that the muscle has unoccupied motor endplates that can be reinnervated with an increased supply of regenerating nerve fibers; therefore, their presence is a requirement for pursuing nerve transfers. ${ }^{10-12,14}$ In contrast, the presence of MUAPs is evidence that there is spontaneous recovery of the injured nerve, and response to intraoperative nerve stimulation confirms the presence of intact nerve fibers (Video 2).

VIDEO 2. Patient 3. Intraoperative stimulation 3 following femoral nerve decompression showing a strong response in the rectus femoris muscle but a weak response in the vastus medialis and lateralis. Copyright Amy M. Moore. Published with permission. Click here to view.

In such cases, an SETS transfer is performed to preserve and augment the regenerating native motor nerve. In cases in which MUAPs and response to intraoperative stimulation are absent, an ETE transfer is performed. Our treatment algorithm is summarized in Fig. 7.

\section{Limitations}

The main limitations of this study are its small sample size and retrospective design. The patients in this series also underwent a combination of interventions, namely, femoral nerve decompression in conjunction with nerve transfer(s), making it difficult to isolate the effect of any one of these interventions. Many patients also received multiple nerve transfers including both the sartorius and obturator nerves. Therefore, the findings of this case series should not be construed as conclusive evidence for their effectiveness. However, this is a technique based on principles established at the basic science level and utilized in the upper extremity, and we are encouraged by the significant improvement in function and pain experienced by our patients. We are in the process of building a prospective database that includes more objective outcome measures at defined time intervals, including PROMIS (PatientReported Outcomes Measurement Information System) scores. 


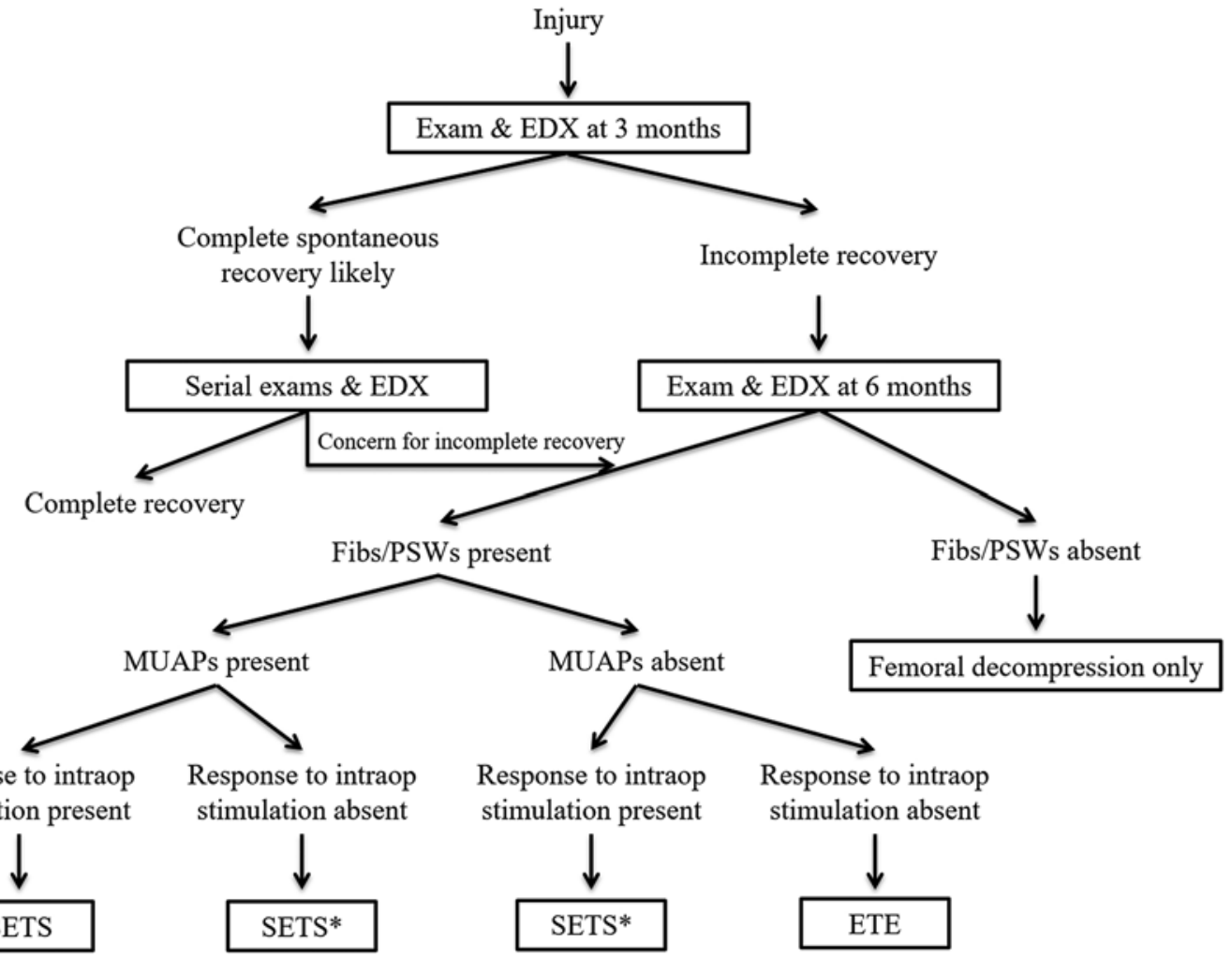

FIG. 7. Our treatment algorithm for partial femoral nerve injuries. EDX = electrodiagnostic studies; fibs = fibrillations; PSW = positive sharp wave. *Electrodiagnostic studies have limitations with regard to the subjectivity and skill of the electrodiagnostician. Intraoperative confirmation with neurophysiology can be used to confirm the recovery and activity of specific muscles.

\section{Conclusions}

Femoral nerve injuries cause significant morbidity, and every effort should be made to restore its function. Branches to the sartorius are often spared in femoral nerve palsies and serve as ideal donors due to their expendability, proximity to the quadriceps branches, and adequate supply of nerve fibers. Furthermore, postoperative outcomes can be maximized by the relatively intuitive donor-activated rehabilitation.

\section{Acknowledgments}

We acknowledge Daniel Hunter for his assistance with histological analysis.

\section{References}

1. Kim DH, Murovic JA, Tiel RL, Kline DG. Intrapelvic and thigh-level femoral nerve lesions: management and outcomes in 119 surgically treated cases. J Neurosurg. 2004;100(6): 989-996.

2. Hallock GG. Restoration of quadriceps femoris function with a dynamic microsurgical free latissimus dorsi muscle transfer. Ann Plast Surg. 2004;52(1):89-92.

3. Ihara K, Shigetomi M, Kawai S, et al. Functioning muscle transplantation after wide excision of sarcomas in the extremity. Clin Orthop Relat Res. 1999;(358):140-148.

4. Kuhlmann RF, Bell JF. A clinical evaluation of tendon transplantations for poliomyelitis affecting the lower extremities. $J$ Bone Joint Surg Am. 1952;34 A(4):915-926.
5. MacArthur IR, McInnes CW, Dalke KR, et al. Patient reported outcomes following lower extremity soft tissue sarcoma resection with microsurgical preservation of ambulation. $J$ Reconstr Microsurg. 2019;35(3):168-175.

6. Pritsch T, Malawer MM, Wu C-C, et al. Functional reconstruction of the extensor mechanism following massive tumor resections from the anterior compartment of the thigh. Plast Reconstr Surg. 2007;120(4):960-969.

7. Willcox TM, Smith AA, Beauchamp C, Meland NB. Functional free latissimus dorsi muscle flap to the proximal lower extremity. Clin Orthop Relat Res. 2003;410(410):285-288.

8. Campbell AA, Eckhauser FE, Belzberg A, Campbell JN. Obturator nerve transfer as an option for femoral nerve repair: case report. Neurosurgery. 2010;66(6)(Suppl Operative): 375 .

9. Tung TH, Chao A, Moore AM. Obturator nerve transfer for femoral nerve reconstruction: anatomic study and clinical application. Plast Reconstr Surg. 2012;130(5):1066-1074.

10. Davidge KM, Yee A, Moore AM, Mackinnon SE. The supercharge end-to-side anterior interosseous-to-ulnar motor nerve transfer for restoring intrinsic function: clinical experience. Plast Reconstr Surg. 2015;136(3):344e-352e.

11. Kale SS, Glaus SW, Yee A, et al. Reverse end-to-side nerve transfer: from animal model to clinical use. J Hand Surg Am. 2011;36(10):1631-1639.e2.

12. Farber SJ, Glaus SW, Moore AM, et al. Supercharge nerve transfer to enhance motor recovery: a laboratory study. $J$ Hand Surg Am. 2013;38(3):466-477.

13. Power HA, Kahn LC, Patterson MM, et al. Refining indications for the supercharge end-to-side anterior interosseous to ulnar motor nerve transfer in cubital tunnel syndrome. Plast Reconstr Surg. 2020;145(1):106e-116e. 
14. Baltzer H, Woo A, Oh C, Moran SL. Comparison of ulnar intrinsic function following supercharge end-to-side anterior interosseous-to-ulnar motor nerve transfer: a matched cohort study of proximal ulnar nerve injury patients. Plast Reconstr Surg. 2016;138(6):1264-1272.

15. Koriem E, El-Mahy MM, Atiyya AN, Diab RA. Comparison between supercharged ulnar nerve repair by anterior interosseous nerve transfer and isolated ulnar nerve repair in proximal ulnar nerve injuries. J Hand Surg Am. 2020;45(2): 104-110.

16. Moore AM, Krauss EM, Parikh RP, et al. Femoral nerve transfers for restoring tibial nerve function: an anatomical study and clinical correlation: a report of 2 cases. J Neurosurg. 2018;129(4):1024-1033.

17. Hunter DA, Moradzadeh A, Whitlock EL, et al. Binary imaging analysis for comprehensive quantitative histomorphometry of peripheral nerve. J Neurosci Methods. 2007;166(1): 116-124.

18. Kahn LC, Moore AM. Donor activation focused rehabilitation approach: maximizing outcomes after nerve transfers. Hand Clin. 2016;32(2):263-277.

19. Tanaka C, Ide MR, Junior AJR. Anatomical contribution to the surgical construction of the sartorius muscle flap. Surg Radiol Anat. 2006;28(3):277-283.

20. Yang D, Morris SF, Sigurdson L. The sartorius muscle: anatomic considerations for reconstructive surgeons. Surg Radiol Anat. 1998;20(5):307-310.

21. Buckland A, Pan WR, Dhar S, et al. Neurovascular anatomy of sartorius muscle flaps: implications for local transposition and facial reanimation. Plast Reconstr Surg. 2009;123(1): 44-54.

22. Yu D-Z, Liu A-T, Dang R-S, et al. Intramuscular innervations of muscle flaps that are commonly used in clinical settings. Surg Radiol Anat. 2010;32(7):637-646.
23. Malawer MM, Kellar-Graney K. Soft tissue reconstruction after limb-sparing surgery for tumors of the upper and lower extremities. Oper Tech Orthop. 2004;14(4):276-287.

\section{Disclosures}

Dr. Moore: Consultant for Checkpoint Surgical, Inc.

\section{Author Contributions}

Conception and design: Moore, McInnes, Power, Tung. Acquisition of data: all authors. Analysis and interpretation of data: all authors. Drafting the article: McInnes, Ha. Critically revising the article: all authors. Reviewed submitted version of manuscript: all authors. Approved the final version of the manuscript on behalf of all authors: Moore. Statistical analysis: Ha, Power. Administrative/technical/material support: Moore, Power. Study supervision: Moore, Tung.

\section{Supplemental Information \\ Videos}

Video 1. https://vimeo.com/440969013.

Video 2. https://vimeo.com/440968782.

\section{Previous Presentations}

This study was previously presented as a podium presentation using Microsoft PowerPoint slides at the Canadian Society of Plastic Surgeons 72nd Annual Meeting, Jasper, Alberta, Canada, June 19-23, 2018.

\section{Correspondence}

Amy M. Moore: The Ohio State University, Columbus, OH. amy.m.moore@osumc.edu. 